J. Clin. Chem. Clin. Biochem.

Vol. 15, 1977, pp. 371-374

\title{
Über das Vorkommen von Purindesoxyribosiden im Harn
}

\author{
Von Margit Solle \\ Staatliches Amt für Atomsicherheit und Strahlenschutz der Deutschen Demokratischen Republik, Berlin-Karlshorst
}

(Eingegangen am 15. Juni 1976/15. Februar 1977)

Zusammenfassung: Die vorliegende Arbeit beschreibt Versuchsergebnisse, die den Nachweis erbringen, daß auch Purindesoxyriboside zu den normalen Kataboliten im Humanharn gehören. Bei gesunden Probanden beträgt die innerhalb 24 Stunden ausgeschiedene Menge etwa $25 \mu \mathrm{g}$ bzw. $41 \%$ der Gesamtdesoxyriboside des Harns. Die Bestimmung der Purindesoxyriboside erfolgte als säurelabiler Anteil der Gesamtdesoxyriboside im bakteriologischen Test mittels Lactobacillus acidophilus R 26.

\section{Occurrence of purine deoxyribosides in urine}

Summary: It was shown that purine deoxyribosides are normal constituents of human urine. Healthy probands excrete about $25 \mu \mathrm{g}$ ( $41 \%$ of the total urinary deoxyribosides) per $24 \mathrm{~h}$. Purine deoxyribosides were determined as the acid-labile fraction of the total deoxyribosides by the microbiological assay with Lactobacillus acidophilus $\mathrm{R} 26$.

\section{Einleitung}

Die Ausscheidung von Desoxyribosiden als Intermediäroder Endprodukte des DNA-Abbaus wurde bisher im wesentlichen unter strahlenbiologischem Aspekt untersucht. 1958 beschrieben Pařizek et al. (1) nạch RöntgenBestrahhlung von Ratten eine dosisabhängige Erhöhung der Exkretion Dische-positiver Substanzen, als deren Hauptkomponente sie Desoxycytidin identifizierten. Seitdem wurden eine Vielzahl von Arbeiten über die Ausscheidung von Desoxyribosiden unter physiologischen Bedingungen sowie nach Einwirkung ionisierender Strahlung bei verschiedenen Tierspezies und beim Menschen publiziert (Ubersichten 1. c. $(2-5)$ ). Im Unterschied zur Ratte, bei dei durch die niedrige Aktivität an Nucleoosid-Aminohydrolase Desoxycytidin dạs dominierende Desóxyribosid im Harn ist (zusammen mit Methyldesoxycytidin beträgt der Anteil etwa 90\% der Gesamtdesoxyriboside (5)), werden im Humanham die drei Pyrimidindesoxyriboside dCyd, dThd und dUrd in nahezu gleicher Konzentration von je 10-20 $\mu \mathrm{g} / \mathrm{d}$ ausgeschieden (6). Uber die Ausscheidung von Purindesoxyribosiden sind uns keine Veröffentlichuingen bekannt.

Die im Vergleich zu ānderen harnpflichtigen Purinund Pyrimidinmetaboliten sehr geringe Konzentration der Desoxyriboside führt dazu, daß diese bei den üblichen Fraktionierungs: und Bestimmungsverfahren nicht erfaßt werden (7-10). Diese Tatsache mag auch die Hauptursache dafür sein, daß in Lehr- und Handbüchern sowie in der einschlägigen Literatur mit Ausnahme der strahlenbiologischen Arbeiten nahezu keine Angaben über Vorkommen, Herkunft und Konzentration der genannten Verbindungen unter normalen und pathologischen Bedingungen $\mathrm{zu}$ finden sind.

Bei der Testung einer Methode zur Bestimmung der Gesamtdesoxyriboside im unfraktionierten Humanharn mittels Lactobacillus acidophilus $\mathrm{R} 26$, einer desoxyribosid-abhängigen Mangelmutante, beobachteten wir, daß die erhaltenen Werte teilweise beträchtlich von der Summe der einzeln bestimmten Pyrimidindësoxyriboside nach dem Verfahren von Gerber et al. (11) oder Blažek et al. (12) abwichen. Mit Verlusten bei den Fraktionierungsverfahren allein ließ sich diese Diskrepanz kaum erklären. Mit größerer Wahrscheinlichkeit war eine Förderung des Lactobacillus-Wachstums durch andere desoxyribosidhaltige oder unspezifisch wirkende Metaboliten anzunehmen. Wir prüften deshalb die Veränderung der Desoxyribosidkonzentration im

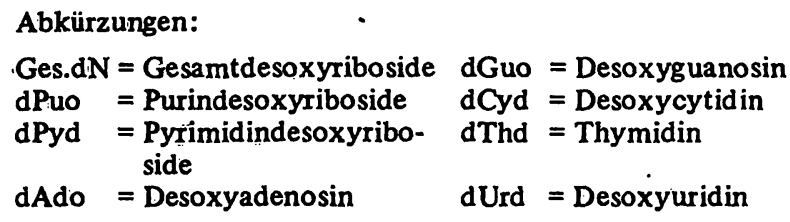


Harn nach Hydrolyse unter Bedingungen, die zur Spaltung der Purindesoxyriboside führen (13). Im Folgenden beschreiben wir Ergebnisse, die auf das Vorkommen von Purindesoxyribosiden im Ham hinweisen.

\section{Material und Methoden}

Die Untersuchungen wurden - soweit nicht anders angegeben mit Harn gesunder Probanden durchgeführt.

Der unter Toluolabschluß gesammelte 24-Stunden-Harn wurde im gefrorenen Zustand aufbewahrt. $10 \mathrm{ml}$ Harn wurden nach dem Aufkochen und Abfiltrieren mit $1 \mathrm{~mol} / 1$ Salzsäure auf $\mathrm{pH}=2,0$ eingestellt und mit dest. Wasser auf $11 \mathrm{ml}$ aufgefültt. $5 \mathrm{ml}$ davon wurden $5 \mathrm{~min}$ im siedenden Wasserbad zur Hydrolyse der Purindesoxyriboside erhitzt, $5 \mathrm{ml}$ dienten zur Vergleichsbestimmung der Gesamtdesoxyriboside. Beide Proben wurden vor der Desoxyribosidbestimmung mit $1 \mathrm{~mol} / 1 \mathrm{KOH}$ auf $\mathrm{pH}=6,5$ eingestellt und mit dest. Wasser auf $6 \mathrm{ml}$ aufgefült.

Die Desoxyribosidbestimmung erfolgte im bakteriologischen Test mittels Lactobacillus acidophilus R 26 (13) unter Verwendung eines synthetischen Nährmediums (2) in einer eigenen, zur Direktbestimmung im Harn geeigneten Modifikation (14). Bei diesem Test zeigen alle natürlichen Desoxyriboside in äquimolaren Mengen den gleichen Wachstumseffekt, da durch eine bakterieneigene Trans-N-Gly cosidase (EC 2.4.2.6) aus einem einzigen Desoxyribosid die anderen zur DNA-Synthese benötigten gebildet werden können (15). 0,1-0,3 ml der Harnproben wurden mit $0,3 \mathrm{ml}$ Medium nach dem Beimpfen mit Lactobacillus acidophilus 16 Stunden bei $37^{\circ} \mathrm{C}$ bebrütet. Das Bakterienwachstum wurde als Trübung bei $650 \mathrm{~nm}$ photometrisch gemessen und mit Hilfe einer Standardkurve (Desoxycytidin als Standardsubstanz) in die entsprechende Desoxyribosid-Konzentration umgerechnet (14).

In einigen Fällen wurde eine papierchromatographische Trennung der einzelnen Desoxyriboside vorgenommen. Unter Verwendung des Papiers Whatman 3 MM $(23 \times 37 \mathrm{~cm})$ wurde im absteigenden Verfahren mit folgenden Laufmittelsystemen und -zeiten gearbeitet:

I n-Butanol, wassergesättigt, Ammoniakatmosphäre $18 \mathrm{~h}$ II sec Butanol, wassergesättigt

$16 \mathrm{~h}$

III tert Butanol-Methylethylketon-Wasser-Ameisen-

säure (Volumina, $44 \mathrm{ml}+44 \mathrm{ml}+11 \mathrm{ml}+0,26 \mathrm{ml}$ ) $6 \mathrm{~h}$

Der Harn wurde nach dem Filtrieren direkt auf das Papier aufgetragen (10 ml/4 Bogen Papier). Die den mitgelaufenen Standard-Desoxyribosiden entsprechenden Streifen der 4 Chro matogramme wurden gemeinsam mit Methanol eluiert und nach Eindampfen zur Trockne die Rückstände in $2-5 \mathrm{ml}$ dest. Wasser aufgenommen. In dieser Lösung erfolgte die bakteriologische Desoxyribosid-Bestimmung direkt oder nach Hydrolyse der Purindesoxyriboside.

\section{Ergebnisse und Diskussion}

Qualitativer und quantitativer Nachweis der Purindesoxyriboside

Bei allen bisher untersuchten Urinproben konnte nach Säurehydrolyse $\left(\mathrm{pH} 2,0,5 \mathrm{~min}, 96^{\circ} \mathrm{C}\right)$ eine deutliche Abnahme der mit dem Lactobacillus-Test erfaßbaren Harnbestandteile nachgewiesen werden. Die entsprechenden Werte zeigt Tabelle 1.

Wegen der bekannten Säurelabilität der Purindesoxy. riboside und entsprechend den Angaben von.HoffJ $\phi$ rgensen (13) müßte die Konzentration nach der Hy-
Tab. 1. Desoxyriboside im Harn ausgewählter gesunder Probanden vor und nach Hydrolyse.

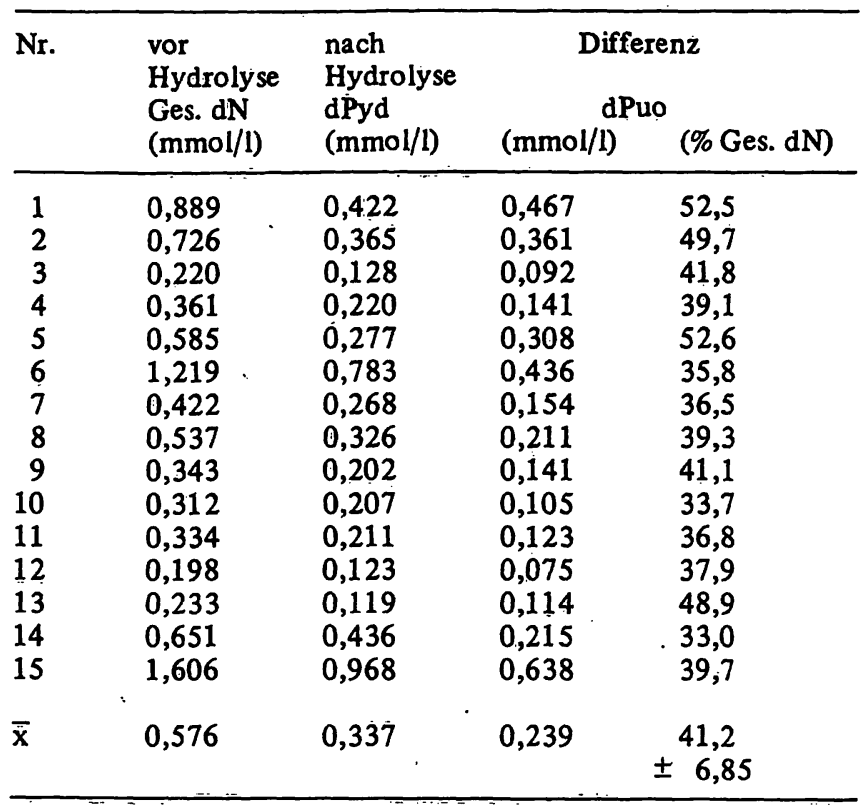

drolyse, die $58,8 \%$ der Gesamtdesoxyriboside beträgt, den Pyrimidindesoxyribosiden und der Differenzbetrag zum Ausgangswert von $41,2 \%$ den Purindexoxyribosiden entsprechen. Für die vorliegenden Untersuchungen wurden nur Harnproben mit einer Desoxyribosidkonzentration oberhalb $0,20 \mathrm{mmol} / 1$ ausgewählt, da bei $0,08 \mathrm{mmol} / 1$ die Nachweisgrenze der verwendeten Direktmethode liegt. Deshalb ist der in Tabelle 1 angegebene Mittelwert für die Konzentrationen nicht repräsentativ für ein Normalkollektiv. Da aber im untersuchten Bereich $(0,2-1,6 \mathrm{mmol} / \mathrm{l})$ der prozentuale Anteil der Purindesoxyriboside konstant ist, erscheint uns eine Extrapolation für den gesamten Normalbereich erlaubt. Die mittlere Konzentration der Gesamtdesoxyriboside im Harn gesunder Probanden $(n=124)$ beträgt $0,309 \pm 0,171 \mathrm{mmol} / 1 \mathrm{bzw} .60 \mu \mathrm{g} / \mathrm{d}$, woraus bei einem Anteil von durchschnittlich $41 \%$ für die Purindesoxyriboside ein Wert von $0,130 \mathrm{mmol} / 1 \mathrm{bzw} .25 \mu \mathrm{g} / \mathrm{d}$ resultieren würde.

Da es sich beim Ham um ein sehr komplexes Gemisch von Stoffwechselprodukten handelt, mußte ausgeschlossen werden, daß die beim Erhitzen im sauren Milieu auftretende Abnahme des Lactobacillus-Wachstums nicht auf eine anderweitige Reaktion von Pyrimidindesoxyribosiden (Komplexbildung oder Zerstörung) oder den Abbau von Hemmstoffen für den verwendeten Testorganismus zurückzuführen ist. Deshalb wurden die verschiedenen Desoxyriboside in einer Konzentration von $0,45 \mu \mathrm{mol} / 1$ einzeln zu Harn zugesetzt und der Lactobacillus:Test vor und nach Hydrolyse durchgeführt. Bei den Kontrollen (unhydrolysierte Proben) betrug die Wiederfindung aller zugesetzten Desoxyriboside 90-110\%. Nach Hydrolyse ( $5 \mathrm{~min}$ ) wurden die drei Pyrimidindeșoxyriboside innerhalb der oben genannten Streubreite wiedergefunden, während die zugesetzten Purindesoxy- 
riboside nicht mehr nachweisbar waren. Bei längerem Erhitzen unter den genannten Bedingungen unterliegt allerdings auch Desoxycytidin erwartungsgemäß einer hydrolytischen Spaltung und damit einer Abnakme seiner Verwertbarkeit durch Lactobacillus. Nach $60 \mathrm{~min}$ Hydrolysezeit betrug die Desoxycytidin-Wiederfindung nur noch etwa $25 \%$. Diese Tatsache bietet auch die Erklärung für den Verlauf der Hydrolysekurven des Harns, die nach $5 \mathrm{~min}$, d. h. nach völliger Spaltung der Purindesoxyriboside, nicht in ein Plateau übergehen, sondern allmählich weiter abfallen (Abb. 1).

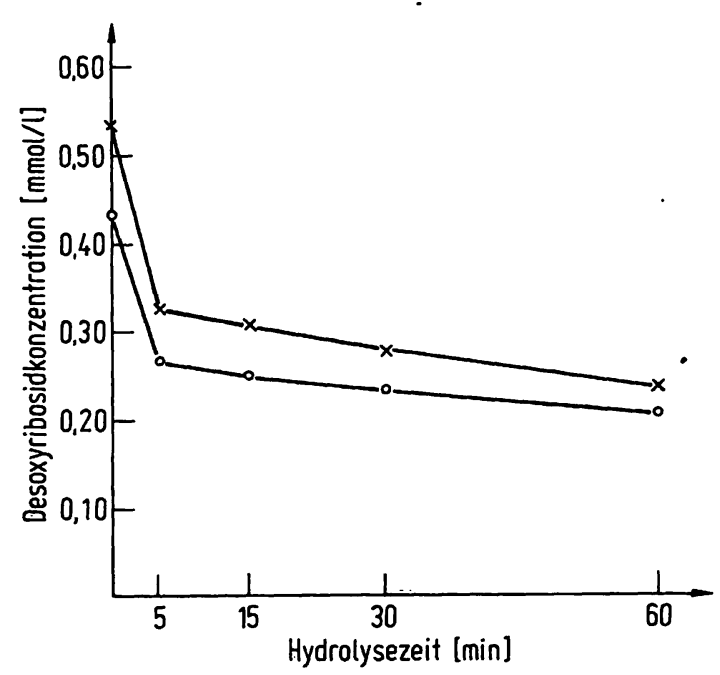

Abb. 1. Abnahme der Desoxyribosidkonzentration bei Säurehydrolyse von 2 Harnen (Probanden $7(0-0)$ und 8 $(x-x)$ aus Tab. 1$)$.

\section{Identifizie rung der Purindesoxy riboside}

Wegen der geringen Konzentration der Harndesoxyriboside ist die völlige Isolierung der einzelnen Komponenten und ihre Identifizierung mit Hilfe der UV-Spektren kaum möglich. Deshalb wurde im vorliegenden Fall ebenso wie bei der Charakterisierung der Pyrimidindesoxyriboside des Harns auf den Vergleich der Wanderungsgeschwindigkeiten bei papierchromatographischer $\bar{T}$ rennung zurückgegriffen. Für diese Untersuchungen wurden einige Harne mit sehr hoher Desoxyribosidkonzentrātion ausgewählt. Da bei einer Reinigung des Harns mittels Dowex-Säulen, wie sie von B̈lažek et al. (12) zur getrẹnnten Erfassung der Pyrimidindesoxyriboside angèwendet wird, Desoxyadenosin und Desoxyguanosin nicht in einer im Lactobacillus-Test nachweisbaren Form wiederzufinden wären, wurde auf dièse Prozedur verzichtet und der Harn direkt auf das Chromatographiepapier aufgetragen. Die Harnproben wurden jeweils in 3 verșchiedenen Laufmittelsystemen, einem neutralen, einem alkalischen und einem sauren, chromatographiert.

Tabelle 2 gibt eine Ưbersicht über die mittleren $\mathbf{R}_{\mathbf{F}^{-}}$ Werte der getesteten Desoxyriboside in den verwendeten Laufmittelsystemen. Daraus ist ersichtlich, daß beim Vorliegen eines Gemisches mit keinem System eine
Tab. 2. $R_{F}$-Werte der einzelnen Desoxyriboside Laufmittelsysteme s. Material und Methoden.

\begin{tabular}{llll}
\hline $\begin{array}{l}\text { Desoxy- } \\
\text { ribosid }\end{array}$ & I & II & III \\
\hline dAdo & 0,76 & 0,75 & 0,45 \\
dGuo & 0,12 & 0,55 & 0,30 \\
dCyd & 0,55 & 0,57 & 0,25 \\
dThd & 0,53 & 0,86 & 0,76 \\
dUrd & 0,25 & 0,74 & 0,63 \\
\hline
\end{tabular}

völlige Trennung aller Desoxyriboside zu erreichen ist, so daß sie meistens paarweise eluiert werden.

Durch Differenzbildungen zwischen den Ergebnissen der Trennung mit verschiedenen Laufmittelsystemen sowie der Desoxyribosidbestimmung vor und nach Hydrolyse konnten Rückschlüsse auf die Lage und damit auch auf die Art der Purindesoxyriboside gezogen sowie eine annähernd quantitative Bestimmung der einzelnen im Harn vorkommenden Desoxyriboside durchgeführt werden.

Bei der qualitativen Beurteilung der Chromatogramme war in allen Eluaten aus den Zonen, die den Standardsubstanzen Desoxyadenosin und Desoxyguanosin entsprachen, ein Lactobacillus-Wachstum nachweisbar, das nach Hydrolyse ganz oder teilweise (je nach Fraktionierungserfolg) verschwand. Desoxyinosin, das als Desaminierungsprodukt des Desoxyadenosins eventuell auch im Harn vorkommen könnte, stand uns in Reinsubstanz nicht zur Verfügung. Nach Literaturangaben $(15,16)$ ist es mit den verwendeten Laufmittelsystemen aber auch kaum von Desoxyguanosin abzutrennen.

Die quantitative Verteilung der Desoxyriboside bei 4 unterschiedlichen Harnen zeigt Tabelle 3. Die Angaben stellen die Mittel aus je 4-10 Werten nach chromatographischer Trennung mit verschiedenen Laufmittelsystemen dar. Die Harne 15 und 21 stammten von gesunden Probanden, 103 von einem Patienten mit Blasentumor vor (A) und nach (B) Bestrahlung. Bei diesem Patienten fanden wir zusätzlich eine Lactobacillus-positive Fraktion auf der Startlinie, die nur mit Wasser, nicht aber mit Methanol eluierbar war, und deren Vorhandensein die vor allem bei 103 B beträchtliche Differenz zwischen der Summe der einzelnen Desoxyriboside und der im Direktverfahren bestimmten Gesamtdesoxyriboside erklärt.

Das prozentuale Verhältnis von Purin- zu Pyrimidindesoxyribosiden entspricht nach chromatographischer Trennung und Summierung der Werte der einzelnen Desoxyriboside dem nach Hydrolyse des unfraktionierten Harns. Zur genaueren Bestimmung des durchschnittlichen Anteils der einzelnen Purin- und Pyrimidindesoxyriboside sind noch weitere Versuche erforderlich. 
Tab. 3. Absolute und relative Konzentration der einzelnen Desoxyriboside in 4 verschiedenẹn Harnen.

\begin{tabular}{|c|c|c|c|c|c|c|c|c|}
\hline $\begin{array}{l}\text { Nr. } \\
\text { Desoxyribosid }\end{array}$ & $\begin{array}{l}15 \\
(\mathrm{mmol} / \mathrm{l})\end{array}$ & $(\%$ von $\Sigma)$ & $\begin{array}{l}21 \\
\text { Konzentration } \\
(\mathrm{mmol} / \mathrm{l})\end{array}$ & $\begin{array}{l}\text { n } \\
(\% \text { von } \Sigma)\end{array}$ & $\begin{array}{l}103 \mathrm{~A} \\
(\mathrm{mmol} / \mathrm{l})\end{array}$ & $(\%$ von $\Sigma)$ & $\begin{array}{l}103 \mathrm{~B} \\
(\mathrm{mmol} / \mathrm{l})\end{array}$ & $(\%$ von $\Sigma)$ \\
\hline $\begin{array}{l}\text { dAdo } \\
\text { dGuo } \\
\text { dCyd } \\
\text { dThd } \\
\text { dUrd }\end{array}$ & $\begin{array}{l}0,224 \\
0,365 \\
0,202 \\
0,255 \\
0,563\end{array}$ & $\begin{array}{l}13,9 \\
22,7 \\
12,6 \\
15,8 \\
35,0\end{array}$ & $\begin{array}{l}0,220 \\
0,070 \\
0,163 \\
0,070 \\
0,216\end{array}$ & $\begin{array}{r}29,8 \\
9,5 \\
22,0 \\
9,5 \\
29,2\end{array}$ & $\begin{array}{l}0,110 \\
1,804 \\
0,572 \\
0,295 \\
1,364\end{array}$ & $\begin{array}{r}2,7 \\
43,5 \\
13,8 \\
7,1 \\
32,9\end{array}$ & $\begin{array}{l}0,277 \\
2,112 \\
0,334 \\
1,478 \\
1,408\end{array}$ & $\begin{array}{r}4,9 \\
37,6 \\
6,0 \\
26,4 \\
25,1\end{array}$ \\
\hline $\begin{array}{l}\Sigma \\
\text { dPuo } \\
\text { dPyd }\end{array}$ & 1,609 & $\begin{array}{r}100,0 \\
36,6 \\
63,4\end{array}$ & 0,739 & $\begin{array}{r}100,0 \\
39,3 \\
60,7\end{array}$ & 4,145 & $\begin{array}{r}100,0 \\
46,2 \\
53,8\end{array}$ & 5,609 & $\begin{array}{r}100,0 \\
42,5 \\
57,5\end{array}$ \\
\hline Ges. dN $\left.{ }_{\text {best. }}{ }^{1}\right)$ & 1,606 & . & 0,651 & & $(4,84)$ & & $(18,48)$ & \\
\hline
\end{tabular}

1) im Direktverfahren bestimmte Gesamtdesoxyriboside

\section{Schlußfolgerung}

Im menschlichen Urin wird neben den bereits mehrfach beschriebenen Pyrimidindesoxyribosiden auch ein nicht unbeträchtlicher Anteil an Purindesoxyribosiden ausgeschieden. Durch papierchromatographische Fraktionierung konnte mit Sicherheit Desoxyadenosin nachge-

\section{Literatur}

1. Pařizek, J., Arient, M., Dienstbier, Z. \& Skoda, J. (1958), Nature (Lond.) 182, 721-722.

2. Arient, M., Dienstbier, Z. \& Shejbal, J. (1967), Acta Univ. Carol. Med. 13, 3-52.

3. Chen, I.-W., Kereiakes, J. G., Friedman, B. I. \& Saenger, E. L. (1968), Radiology 91, 343-348.

4. Fedorova, T. A. (1971), in „Biochemical Indicators of Radiation Injury in Man". International Atomic Energy Agency, Vienna STI/PUB/280 S. 61-74.

5. Solle, M. (1972), Report SZS-147 (Report der Staatlichen Zentrale für Strahlenschutz der DDR).

6. Dienstbier, Z. \& Buřičc, L. (1975), Strahlentherapie 149, 208-211.

7. Adams, W. S., Davis, F. \& Nakatani, M. (1960), Am. J. Med. 28, 726-734.

8. Heirwegh, K. P. M., Ramboer, C. \& de Groote, J. (1967), Am. J. Med. 42, 913-922. wiesen werden. Eine zweite Fraktion entspricht aufgrund ihrer $R_{F}$-Werte Desoxyguanosin und/oder Desoxyinosin. Ziel dieser Mitteilung soll es zunächst nur sein, auf diese Tatsache aufmerksam zu machen. In weiteren Untersuchungen wird geprüft, ob sich nach Einwirkung ionisierender Stahlung das quantitative Verhältnis zwischen purin- und pyrimidinhaltigen Desoxyribosiden verändert.
9. Fink, K. \& Adams, W. S. (1968), Arch. Biochem. Biophys. 126, 27-33.

10. Scott, C. D. (1968), Clin. Chem. 14, 521-528.

11. Gerber, G. B., Zicha, B., Mathieu, D., Remy-Defraigne, J. \& Deroo, J. (1970), in „Biochemisch nachweisbare Strahlenwirkungen und deren Beziehungen zur Strahlentherapie“". (Gerber, G. B. ed.) G. Thieme Verlag, Stuttgart, S. $40-44$.

12. Blažek, L., Arient, M., Budešinsky, M., Kovał̌ikova, M., Bufic, L. \& Dienstbier, Z. (1972), Collect. Czech. Chem. Commun. 37, 1933-1937.

13. Hoff-J $\phi$ rgensen, E. (1952), Biochem. J. 50, 400-403

14. Solle, M. \& Gensicke, F. (1976), Z. Med. Labortechnik $17,336$.

15. MacNutt, W. S. (1952), Biochem. J. 50, 384-397.

16. Fink, K. \& Adams, W. W. (1966), J. Chromatog. 22, 118-129.
Dr. rer. nat. Margit Solle Staatliches Amt für Atomsicherheit und Strahlenschutz der DDR Waldowallee 117 DDR-1157 Berlin 